\title{
Unsuitability of the moving light clock system for the Lorentz factor derivation
}

\author{
Tomasz T. Wałek* \\ Department of Environmental and Safety Management, \\ Silesian University of Technology, 66 De Gaulle St., 41-800 Zabrze, Poland
}

\begin{abstract}
The moving light clock system was analyzed with respect to the orientation of the wavefront of the light pulse observed in the moving and stationary frames of reference. The plane wavefront of the light pulse was oriented horizontally in both the frames. The wavefront observed in the stationary frame was not perpendicular to the direction of the light pulse propagation. This showed different characteristics of the light pulse than that assumed in the Lorentz factor derivation. According to the horizontal orientation of the wavefront, velocity $c$ was determined as the vertical component of the light pulse motion observed in the stationary frame. Application of this velocity distribution in the Lorentz factor derivation showed the same travel time for the light pulse observed in the moving and stationary frames of reference. The moving light clock system was therefore found to be unsuitable for the Lorentz factor derivation and illustration of time dilation, and shown to illustrate the relativity of the observation of light rather than the relativity of time.
\end{abstract}

\section{INTRODUCTION}

The Lorentz factor plays a fundamental role in relativistic calculations. It can be derived in several ways $[1,2$, 3, 4, 5, 6, 7, 8, 9, 10, 11, 12, 13, 14], nevertheless, the method of the moving light clock [15] is used in many textbooks and relativity courses. Therefore, it is important to analyze all aspects of the applicability of this method.

Two frames of reference are considered in this derivation. Frame $S$ is stationary and frame $S^{\prime}$ moves along the $x$-axis of frame $S$ with uniform velocity $v$. The light clock is arranged vertically in frame $S^{\prime}$ (Fig. 1a). It consists of two mirrors between which the light pulse emitted in frame $S^{\prime}$ travels in vacuum back and forth. An observer in frame $S^{\prime}$ observes the distance traversed by the light pulse between the mirrors as $y^{\prime}$ and the travel time as $t^{\prime}$. An observer in frame $S$ (Fig. 1b) observes the distance as $d$ and the time as $t$. According to the postulate of invariance of the speed of light, the light pulse travels in vacuum with velocity $c$ regardless of the frame of reference. Thus, distance $y^{\prime}$ can be expressed as $c t^{\prime}$ and distance $d$ as $c t$. Since distance $d$ is greater than $y^{\prime}$ then $c t>c t^{\prime}$ and hence $t>t^{\prime}$. This means that the time

(a)

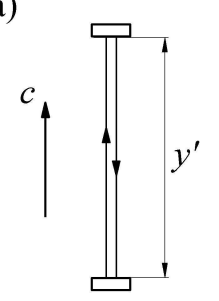

(b)

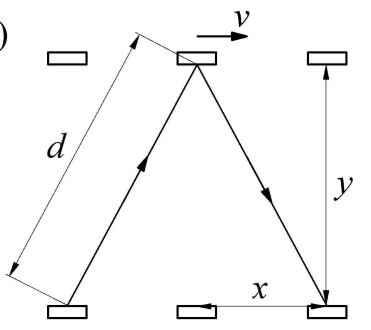

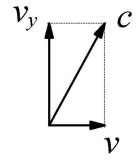

Figure 1: The moving light clock observed in the moving (a) and stationary (b) frame of reference.

*Electronic address: tomasz.walek@polsl.pl in which the light pulse travels the distance between the mirrors is longer in frame $S$ than in frame $S^{\prime}$. By equating distances $y^{\prime}$ and $y$ we have $c t^{\prime}=\sqrt{(c t)^{2}-(v t)^{2}}$ and we can calculate the Lorentz factor $\gamma=1 / \sqrt{1-v^{2} / c^{2}}$.

This paper is intended to analyze the moving light clock system in relation to the orientation of the wavefront of the light pulse observed in the moving and stationary frames of reference.

\section{ORIENTATION OF THE WAVEFRONT OF THE LIGHT PULSE IN THE MOVING LIGHT CLOCK SYSTEM}

The light pulse applied in the moving light clock system travels the distance between the mirrors without dispersion and remains unchanged in form. It means that the light of the light pulse is collimated. Therefore, the light pulse traveling between the mirrors in vacuum can be characterized by a plane wavefront which is perpendicular to the wave normal and to the direction of the light pulse propagation. This characteristic of the light corresponds to a laser beam and hence the light pulse applied in the light clock is usually described as a laser pulse. According to the principle of relativity, this characteristics of the light pulse must be valid in any inertial reference frame.

The light pulse observed in frame $S^{\prime}$ travels vertically between the mirrors and its wavefront is parallel to the surface of the mirrors (Fig. 2a). The horizontally oriented wavefront will also be observed horizontally in frame $S$ (Fig. 2b), because the speed of relative motion of frame $S^{\prime}$ is zero in the direction $y$. Hence, coordinates $y^{\prime}$ and $y$ of any point of the wavefront must have the same values as observed in both the reference frames. From the same reason, the horizontal orientation of the mirrors is not influenced by the motion of frame $S^{\prime}$. (See Appendix for additional discussion of the orientation of the wavefront.)

It can be seen that the orientation of the wavefront of the light pulse observed in frame $S$ is not perpendicu- 
(a)

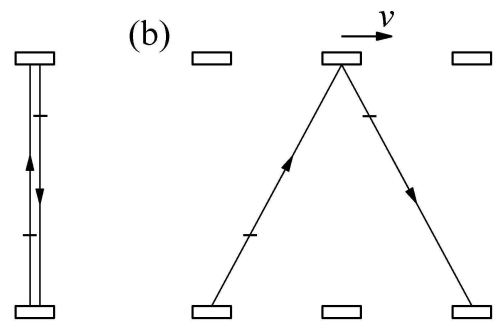

Figure 2: The orientation of the wavefront of the light pulse in the moving light clock system observed in the moving (a) and stationary (b) frame of reference. (a)

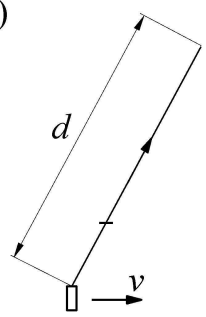

(b)

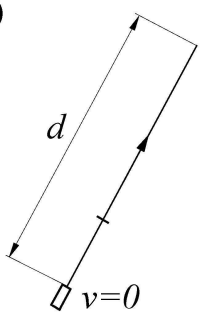

Figure 3: The orientation of the wavefront of the light pulse emitted in the moving light clock system (a) and in the stationary frame of reference (b), as observed in the stationary frame.

lar to the direction of the light pulse propagation (Fig. 3a). The light pulse therefore does not correspond to the characteristics of laser light traveling in vacuum. Such a situation, where the wavefront of the light pulse is perpendicular to the direction of propagation, occurs for the light pulse emitted in the stationary frame of reference along path $d$, independently of the moving light clock system (Fig. 3b). In both the cases the orientation of the wavefront is determined by the orientation of the emitter during emission. The distances traveled in both cases are the same, but the light pulses cannot be considered identical because of the different wavefront orientations.

This shows the complex character of the light pulse motion observed in the moving light clock system in frame $S$. In this case the motion of the light pulse cannot be treated as an independent motion. It is a visual summation of two independent motions - the light pulse moving in relation to frame $S^{\prime}$ and frame $S^{\prime}$ moving in relation to frame $S$. Therefore, it cannot be said that the light pulse travels distance $d$ in frame $S$ with velocity $c$, as it is assumed in the Lorentz factor derivation, because the light pulse observed in frame $S$ does not correspond to the characteristics of the light pulse traveling in vacuum. We can only state that the motion of the light pulse and the motion of frame $S^{\prime}$ form in frame $S$ an image of the light pulse characterized by the wavefront oriented not perpendicularly to the direction of propagation, and moving along path $d$. (a)

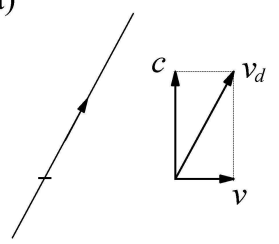

(b)

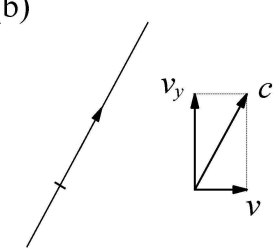

Figure 4: The velocity distribution of the light pulse emitted in the moving light clock system (a) and in the stationary frame of reference (b), as observed in the stationary frame.

\section{VELOCITY DISTRIBUTION IN THE MOVING LIGHT CLOCK SYSTEM}

Since the plane wavefront of the light pulse traveling in vacuum is perpendicular to the direction of propagation, and the light pulse propagates in vacuum with velocity $c$, this means that velocity $c$ is perpendicular to the wavefront of the light pulse. It shows that the orientation of velocity $c$ is closely related to the orientation of the wavefront. Because of the principle of relativity, this relation must be valid in any inertial reference frame.

For the moving light clock system observed in frame $S$, this means that velocity $c$ is the vertical component of the motion of the light pulse (Fig. 4a). The component velocity $v$ is in this case not related to the motion of the light pulse. It is a result of the movement of frame $S^{\prime}$ in relation to frame $S$ only, and any change in velocity of frame $S^{\prime}$ will affect it.

This velocity distribution differs from the assumptions of the Lorentz factor derivation, where velocity $c$ is supposed to occur along path $d$ (Fig. 1b). Such a situation, where velocity $c$ is oriented along path $d$ and is perpendicular to the wavefront of the light pulse, occurs for the light pulse emitted in the stationary frame of reference, independently of the moving light clock system (Fig. 4b). In this case the component velocity $v$ is related to the motion of the light pulse only - no movement of any reference frame in relation to frame $S$ can influence it.

\section{DISCUSSION}

The analysis of the orientation of the wavefront of the light pulse in the moving light clock system shows that the horizontally oriented wavefront observed in frame $S^{\prime}$ will also be observed horizontally in frame $S$ (Fig. 2). The light pulse observed in the stationary frame is characterized by the wavefront oriented not perpendicular to the direction of propagation. This shows the complex character of the light pulse motion observed in frame $S$. The motion observed in frame $S$ along path $d$ is a visual summation of the motions of the light pulse in relation to frame $S^{\prime}$ and frame $S^{\prime}$ in relation to frame $S$.

According to the horizontal orientation of the wavefront in frame $S$ it can be seen, that the velocity distri- 
bution with vector $c$ oriented vertically (Fig. $4 \mathrm{a}$ ) should be applied in the Lorentz factor derivation. In this case we have $c t^{\prime}=\sqrt{\left(v_{d} t\right)^{2}-(v t)^{2}}$, which leads to $t^{\prime}=t$. It means that the travel time of the light pulse is the same in the moving and stationary frames of reference. The occurrence of velocity $v_{d}$ greater than $c$ does not mean exceeding the speed of light by the light pulse in this case, because the motion observed in frame $S$ along path $d$ is not an independent motion of the light pulse.

Both the complex character of the observation of the light pulse in frame $S$ and the velocity distribution resulting from the horizontal orientation of the wavefront of the light pulse show that the moving light clock system is unsuitable for the Lorentz factor derivation and illustration of time dilation. The analysis presented in this paper suggests that the moving light clock system presents an argument for the simple emission theory rather than the special relativity theory, and illustrates the relativity of the observation of light rather than the relativity of time.

\section{CONCLUSIONS}

The plane wavefront of the light pulse in the moving light clock system is oriented horizontally both in the moving and stationary frames of reference. The light pulse observed in the stationary frame is characterized by the wavefront oriented not perpendicular to the direction of propagation. It shows different characteristics of the light pulse than that assumed in the Lorentz factor derivation.

The horizontal orientation of the wavefront observed in the stationary frame of reference allows to determine the velocity distribution of the light pulse with vertically oriented velocity $c$. Application of this velocity distribution in the Lorentz factor derivation shows that the travel time of the light pulse is the same in the moving and stationary frames of reference.

The analysis of the moving light clock system in relation to the orientation of the wavefront of the light pulse shows that the moving light clock system is unsuitable for the Lorentz factor derivation and illustration of time dilation. The moving light clock system illustrates the relativity of the observation of light rather than the relativity of time.

\section{Appendix: HORIZONTAL ORIENTATION OF THE WAVEFRONT}

The horizontal orientation of the wavefront observed both in the moving and stationary frames of reference can also be verified as follows.

The moving light clock system can be modified so that selective reflection mirrors are applied, which reflect only the light with a wavefront parallel to the mirror surface. When the modified light clock remains stationary in relation to frame $S$, the observers in both the reference frames agree that the plane wave of the light pulse reaches the mirror parallel to its surface (Fig. 5a). (a)

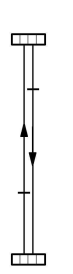

(b)

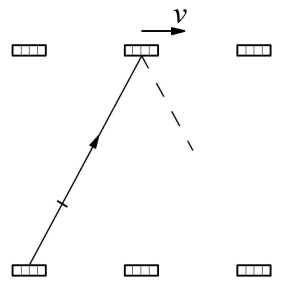

(c)

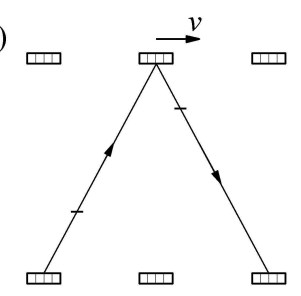

Figure 5: The moving light clock with selective reflection mirrors, as observed in frame $S$ : (a) the light clock remaining stationary in relation to frame $S$; (b) the light clock moving in relation to frame $S$, with the wavefront oriented not horizontally; (c) the light clock moving in relation to frame $S$, with the wavefront oriented horizontally.

In this case the reflection will occur and the observers will observe the light pulse traveling back and forth between the mirrors. However, when the light clock starts moving along the $x$-axis of frame $S$, we can consider two possibilities with regard to the observation of the wavefront in frame $S$ - the first one with the slanted wavefront (Fig. 5b), and the second one with the wavefront oriented horizontally (Fig. 5:).

In the first case, the wavefront of the light pulse reaches the mirror in frame $S$ not parallel to its surface (Fig. 5b), and consequently the reflection cannot occur. This means that any motion of the light clock along the $x$ axis, even with only minimal velocity, should exclude the reflection of the light pulse in frame $S$. At the same time, the observer in frame $S^{\prime}$ will observe the light pulse as reflecting between the mirrors, because the angle of incidence of the light pulse observed in frame $S^{\prime}$ cannot be influenced by any motion of the light clock in relation to frame $S$. Thus, the assumption of the non-horizontal orientation of the wavefront leads to a paradoxical situation where the reflection of the light pulse can be observed in one frame of reference but not in the other.

The exclusion of reflection observed in frame $S$ does not occur in the second case, in which the horizontally oriented wavefront reaches the mirror parallel to the surface (Fig. 吕). In this case the motion of the light pulse and its reflection can be observed in both the reference frames. In fact, the reflection takes place in frame $S^{\prime}$, and in frame $S$ it is only an observation.

Therefore, the plane wavefront observed in the moving light clock system is shown to be oriented horizontally in both the moving and stationary frames of reference. 
[2] Einstein, A.: Relativity: The Special and General Theory, pp. 131-138. Three Rivers Press, New York (1995)

[3] de Felice, F., Sigalotti, L.D.G., Mejias, A.: Lorentz transformations and complex space-time functions. Chaos, Solitons \& Fractals 21, 573-578 (2004)

[4] Fowles, G.R.: Self-inverse form of the Lorentz transformation. Am. J. Phys. 45, 675-676 (1977)

[5] Harvey, A.L.: Lorentz Transformation. Am. J. Phys. 36, 901-904 (1968)

[6] Lee, A.R., Kalotas, T.M.: Lorentz transformations from the first postulate. Am. J. Phys. 43, 434-437 (1975)

[7] Lévy-Leblond, J-M.: One more derivation of the Lorentz transformation. Am. J. Phys. 44, 271-277 (1976)

[8] Macdonald, A.: Derivation of the Lorentz transformation. Am. J. Phys. 49, 493 (1981)

[9] Nadeau, G.: The Lorentz-Einstein Transformation Obtained by a Vector Method. Am. J. Phys. 30, 602-603
(1962)

[10] Pfleiderer, J.: Lorentz Transformation Derived from First-Order Experiments. Am. J. Phys. 37, 1131-1134 (1969)

[11] Schwartz, H.M.: Deduction of the general Lorentz transformations from a set of necessary assumptions. Am. J. Phys. 52, 346-350 (1984)

[12] Sen, A.: How Galileo could have derived the special theory of relativity. Am. J. Phys. 62, 157-162 (1994)

[13] Sigalotti, L.D.G, Mejias, A.: The golden ratio in special relativity. Chaos, Solitons \& Fractals 30, 521-524 (2006)

[14] Srivastava, A.M.: Invariant speed in special relativity. Am. J. Phys. 49, 504-505 (1981)

[15] Feynman, R.P., Leighton, R.B., Sands, M.: The Feynman Lectures on Physics, pp. 15-5-15-6. Addison-Wesley, Reading, Massachusetts (1963) 